ORIGINAL ARTICLE

\section{Levosimendan for Hemodynamic Support after Cardiac Surgery}

\author{
G. Landoni, V.V. Lomivorotov, G. Alvaro, R. Lobreglio, A. Pisano, F. Guarracino, \\ M.G. Calabrò, E.V. Grigoryev, V.V. Likhvantsev, M.F. Salgado-Filho, A. Bianchi, \\ V.V. Pasyuga, M. Baiocchi, F. Pappalardo, F. Monaco, V.A. Boboshko, \\ M.N. Abubakirov, B. Amantea, R. Lembo, L. Brazzi, L. Verniero, P. Bertini, \\ A.M. Scandroglio, T. Bove, A. Belletti, M.G. Michienzi, D.L. Shukevich, \\ T.S. Zabelina, R. Bellomo, and A. Zangrillo, for the CHEETAH Study Group*
}

A BSTRACT

\section{BACKGROUND}

Acute left ventricular dysfunction is a major complication of cardiac surgery and is associated with increased mortality. Meta-analyses of small trials suggest that levosimendan may result in a higher rate of survival among patients undergoing cardiac surgery.

\section{METHODS}

We conducted a multicenter, randomized, double-blind, placebo-controlled trial involving patients in whom perioperative hemodynamic support was indicated after cardiac surgery, according to prespecified criteria. Patients were randomly assigned to receive levosimendan (in a continuous infusion at a dose of 0.025 to $0.2 \mu \mathrm{g}$ per kilogram of body weight per minute) or placebo, for up to 48 hours or until discharge from the intensive care unit (ICU), in addition to standard care. The primary outcome was 30-day mortality.

\section{RESULTS}

The trial was stopped for futility after 506 patients were enrolled. A total of 248 patients were assigned to receive levosimendan and 258 to receive placebo. There was no significant difference in 30-day mortality between the levosimendan group and the placebo group (32 patients [12.9\%] and 33 patients [12.8\%], respectively; absolute risk difference, 0.1 percentage points; $95 \%$ confidence interval [CI], -5.7 to 5.9; $\mathrm{P}=0.97)$. There were no significant differences between the levosimendan group and the placebo group in the durations of mechanical ventilation (median, 19 hours and 21 hours, respectively; median difference, -2 hours; $95 \% \mathrm{CI},-5$ to 1; $\mathrm{P}=0.48$ ), ICU stay (median, 72 hours and 84 hours, respectively; median difference, -12 hours; $95 \% \mathrm{CI},-21$ to $2 ; \mathrm{P}=0.09$ ), and hospital stay (median, 14 days and 14 days, respectively; median difference, 0 days; $95 \% \mathrm{CI},-1$ to $2 ; \mathrm{P}=0.39$ ). There was no significant difference between the levosimendan group and the placebo group in rates of hypotension or cardiac arrhythmias.

\section{CONCLUSIONS}

In patients who required perioperative hemodynamic support after cardiac surgery, low-dose levosimendan in addition to standard care did not result in lower 30-day mortality than placebo. (Funded by the Italian Ministry of Health; CHEETAH ClinicalTrials.gov number, NCT00994825.)
The authors' full names, academic degrees, and affiliations are listed in the Appendix. Address reprint requests to Dr. Bellomo at the Department of Intensive Care, Austin Hospital, Heidelberg, Melbourne, VIC 3084, Australia, or at rinaldo .bellomo@austin.org.au.

*A complete list of investigators in the Levosimendan to Reduce Mortality in High Risk Cardiac Surgery Patients: A Multicenter Randomized Controlled Trial (CHEETAH) Study Group is provided in the Supplementary Appendix, available at NEJM.org.

This article was published on March 21, 2017, and updated on April 12, 2017, at NEJM.org.

N Engl J Med 2017;376:2021-31. DOI: 10.1056/NEJMoal616325

Copyright (๑) 2017 Massachusetts Medical Society. 
$\mathrm{E}$ VERY YEAR, MORE THAN 1 MILLION PAtients undergo cardiac surgery in the United States and Europe. ${ }^{1}$ Acute perioperative left

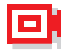

A Quick Take is available at NEJM.org ventricular dysfunction is a major complication affecting up to $20 \%$ of such patients ${ }^{2,3}$ and is associated with increased mortality. ${ }^{4}$ Inotropic drugs (catecholamines and phosphodiesterase type 3 [PDE-3] inhibitors) are the cornerstone of postoperative hemodynamic support. ${ }^{3,5}$ However, no randomized, controlled trials have shown the superiority of any inotropic agent in terms of major clinical outcomes. Furthermore, meta-analyses and observational studies suggest that catecholamines and PDE-3 inhibitors may increase mortality.,

Levosimendan (Simdax, Orion) is an inotropic agent that has been shown to be associated with a higher rate of survival than other inotropic agents in meta-analyses, ${ }^{8}$ especially those involving patients undergoing cardiac surgery. ${ }^{9-11}$ A network meta-analysis ranked levosimendan as the most likely inotrope to reduce mortality among patients undergoing cardiac surgery. ${ }^{12}$ Treatment with levosimendan results in greater cardiac output than does treatment with catecholamines or PDE-3 inhibitors, with minimal effect on myocardial oxygen consumption..$^{13,14}$ Moreover, it has antioxidant, antiinflammatory, and direct cardioprotective effects. ${ }^{13}$ Accordingly, it is widely used in several countries.

Considering the pharmacologic properties of levosimendan and the results of previous studies, we hypothesized that the administration of levosimendan, in addition to standard treatment, might result in lower mortality in this context. Accordingly, we designed the Levosimendan to Reduce Mortality in High Risk Cardiac Surgery Patients: A Multicenter Randomized Controlled Trial (CHEETAH) to test the hypothesis that levosimendan treatment in addition to standard inotropic treatment would result in lower mortality than placebo among patients with perioperative cardiovascular dysfunction after cardiac surgery.

\section{METHODS}

TRIAL DESIGN

We performed this randomized, double-blind, placebo-controlled trial at 14 centers in Italy, Russia, and Brazil. The trial protocol (available with the full text of this article at NEJM.org) was approved by the ethics committee at all the participating centers. Details of the trial methods and statistical analysis plan have been published previously. ${ }^{15}$

The trial was funded by the Italian Ministry of Health and received a start-up grant from the European Association of Cardiothoracic Anesthesiologists. Levosimendan was provided free of charge by the manufacturer (Orion) to centers that recruited patients in Italy; all the centers in Russia and Brazil purchased the drug at full cost. The funders and Orion had no role in the trial design, the data collection and analysis, the writing of the manuscript, or the decision to submit the manuscript for publication. All the authors vouch for the completeness and accuracy of the data and all analyses and for the fidelity of the trial to the protocol.

\section{ENROLLMENT PROCEDURE, CRITERIA, AND RANDOMIZATION}

All the patients who were scheduled for cardiac surgery at the trial centers provided preoperative written informed consent. Patients then underwent randomization if they met the enrollment criteria either in the operating room or in the intensive care unit (ICU). Patients were included if they had perioperative cardiovascular dysfunction, which was defined as the presence of at least one of the following criteria: a preoperative left ventricular ejection fraction of less than $25 \%$, preoperative support with an intraaortic balloon pump, or the need for support with an intraaortic balloon pump or high-dose inotropic support (defined as a vasoactive-inotropic score of $\geq 10$ as described in the Supplementary Appendix, available at NEJM.org) in order to be weaned from cardiopulmonary bypass or at any time within the first 24 hours after surgery.

Exclusion criteria were a previous adverse response to levosimendan, inclusion in another randomized trial, receipt of levosimendan in the previous 30 days, receipt of a kidney or liver transplant, liver cirrhosis, a decision to use extracorporeal membrane oxygenation, or the presence of a do-not-resuscitate order. Patients undergoing an emergency operation were also excluded because it would have been difficult to obtain informed consent.

Eligible patients were randomly assigned, in a 1:1 ratio, to receive either levosimendan or placebo. Randomization was performed with the use of a computer-generated, permuted block sequence 
stratified according to trial center. Trial-group assignments were concealed in sealed, opaque, sequentially numbered envelopes. Physicians, investigators, data collectors, and outcome assessors were unaware of the trial-group assignments.

\section{CLINICAL REGIMEN}

Patients were assigned to receive a blinded infusion of either levosimendan or placebo as prepared by dedicated trial personnel. Levosimendan was diluted as $12.5 \mathrm{mg}$ in $100 \mathrm{ml}$ of $5 \%$ glucose. A mixed-vitamins solution with a yellow color, devoid of relevant cardiovascular effects and indistinguishable in appearance from levosimendan, was used as placebo (see the Supplementary Appendix).

Levosimendan or placebo was initiated as a continuous infusion at a dose of $0.05 \mu \mathrm{g}$ per kilogram of body weight per minute. The dose could then be increased or decreased at the discretion of the attending physician; the minimum dose was $0.025 \mu \mathrm{g}$ per kilogram per minute, and the maximum dose $0.2 \mu \mathrm{g}$ per kilogram per minute. The infusion could be continued for up to 48 hours (to allow for prolonged support in the most compromised patients) or until ICU discharge. In this pragmatic trial, ${ }^{16}$ all clinical decisions, with the exception of the administration of the trial regimen, were left to the discretion of the attending physicians, including hemodynamic monitoring and management. However, an advisory flowchart for open-label inotrope management was provided to investigators (Fig. S1 in the Supplementary Appendix).

\section{DATA COLLECTION AND FOLLOW-UP}

We collected preoperative data on baseline characteristics and coexisting conditions, intraoperative and postoperative treatment data, postoperative laboratory values, duration of mechanical ventilation, durations of ICU and hospital stays, and major outcomes. Baseline hemodynamic data were collected at randomization (most patients were already receiving hemodynamic support with high-dose inotropic agents, an intraaortic balloon pump, or both). We also collected data on the incidence of hypotension, arrhythmias, and other adverse events during the administration of the infusion, as well as information on protocol deviations. Telephone follow-up was performed at 30 days and 180 days after randomization by an investigator who was unaware of the trialgroup assignments.

\section{OUTCOME MEASURES}

The primary outcome of the trial was 30-day mortality. Prespecified secondary outcomes were the following: acute kidney injury, ${ }^{17}$ a need for renal-replacement therapy, a composite outcome of death and need for renal-replacement therapy, duration of mechanical ventilation, and durations of stay in the ICU and hospital. ${ }^{15}$ We also collected data on the following outcomes: need for advanced mechanical circulatory support, myocardial infarction, type 1 or type 2 neurologic damage, ${ }^{18}$ need for tracheostomy, sepsis, pneumonia, and mediastinitis. Definitions of the outcome measures are provided in the Supplementary Appendix. Cause of death in the ICU and hospital was recorded with the use of previously validated criteria (see the Supplementary Appendix). ${ }^{19}$

\section{STATISTICAL ANALYSIS}

The sample-size calculation was based on a twosided alpha error of 0.05 and $80 \%$ power. On the basis of meta-analyses that estimated that mortality would be reduced from $12.7 \%$ to $4.7 \%$ with levosimendan use, ${ }^{9,10}$ we expected $10 \%$ mortality in the placebo group and 5\% mortality in the levosimendan group. Accordingly, we calculated that a sample of 435 patients per group was needed. In order to account for protocol deviations and withdrawal of consent, we planned for 500 patients per group to undergo randomization.

Interim analyses were planned after enrollments of $25 \%$ and $50 \%$ of the sample size. ${ }^{20,21}$ The first review by the data and safety monitoring board (at 25\% enrollment) led to a decision to decrease the sample from 1000 to 500 patients, because a higher-than-expected overall mortality rate of $13.5 \%$ and a lower-than-expected withdrawal rate of $0 \%$ were observed. The second review by the data and safety monitoring board (at $50 \%$ of the originally planned enrollment) led to the decision to stop the trial on the grounds of futility (see the Supplementary Appendix).

Details of the statistical analysis plan have been published previously. ${ }^{15}$ Primary analyses comparing levosimendan with placebo were performed according to the intention-to-treat principle. No imputation for missing data was applied. Per-protocol and as-treated analyses were also performed. 
Data are presented as medians and interquartile ranges for nonnormally distributed variables and as means and standard deviations for normally distributed variables. Dichotomous data (including the primary outcome) were compared by two-tailed chi-square tests with the Yates correction or by Fisher's exact test as appropriate. The primary analysis was not adjusted for covariates. Continuous measurements were compared with the use of the Mann-Whitney U test. A logisticregression model with stepwise selection was used to identify predictors of death (see the Supplementary Appendix). Prespecified subgroup analyses were performed as described in the Supplementary Appendix. In all the subgroup analyses, heterogeneity was estimated by the chi-square test for heterogeneity and the $\mathrm{I}^{2}$ statistic.

All reported P values are two-sided. Data were stored electronically and analyzed with the use of Stata software, version 13 (StataCorp).

\section{RESULTS}

TRIAL POPULATION AND BASELINE CHARACTERISTICS

From November 2009 through April 2016, we obtained written informed consent from 4725 patients. Of these, 506 patients underwent randomization, with 248 patients being randomly assigned to receive levosimendan and 258 to receive placebo (Fig. 1). Most patients underwent randomization in the operating room because high doses of inotropes were indicated for weaning from cardiopulmonary bypass (61 patients [12.1\%]) or in the ICU because of postoperative acute cardiovascular dysfunction (329 [65.0\%]). Only a minority of patients underwent randomization preoperatively because of low ejection fraction (22 patients [4.3\%]). The remaining 94 patients (18.6\%) underwent randomization because they received support with an intraaortic balloon pump. The timing of the randomization of patients who underwent randomization in the ICU is shown in Figure S2 in the Supplementary Appendix. The baseline and intraoperative characteristics of the patients were similar in the levosimendan group and in the placebo group (Table 1, and Table S1 in the Supplementary Appendix).

\section{INFUSION OF LEVOSIMENDAN OR PLACEBO}

The mean $( \pm S D)$ duration of the infusion was $33 \pm 14.6$ hours in the levosimendan group and $32 \pm 13.5$ hours in the placebo group $(\mathrm{P}=0.17)$. The mean dose was $0.066 \pm 0.031 \mu$ g per kilogram per minute in the levosimendan group, with a volume equivalent to a dose of $0.075 \pm 0.033 \mu \mathrm{g}$ per kilogram per minute administered in the placebo group $(\mathrm{P}=0.002)$. An increase from the initial dose of $0.05 \mu \mathrm{g}$ per kilogram per minute was performed in 127 patients (51.2\%) in the levosimendan group, as compared with 159 (61.6\%) in the placebo group ( $\mathrm{P}=0.02)$. Reasons for the interruption of the infusion and for unblinding are reported in Tables S2 and S3, respectively, in the Supplementary Appendix.

\section{HEMODYNAMIC VARIABLES AND PROCESS OF CARE}

Hemodynamic data were not available for all patients, because hemodynamic monitoring was not required and was performed according to the clinical condition of the patient and the judgment of the physician. The available hemodynamic data, the rates of use of vasoactive drugs, and the inotropic score after randomization were similar in the two groups. There was no between-group difference in the available postoperative laboratory values. (Details are provided in Tables S4 through S8 in the Supplementary Appendix.)

\section{PRIMARY AND SECONDARY OUTCOMES}

No patient was lost to 30-day follow-up, and all the patients who underwent randomization were included in the intention-to-treat analysis (Fig. 1). At 30 days, there had been 32 deaths $(12.9 \%$ of patients) in the levosimendan group and 33 deaths $(12.8 \%)$ in the placebo group (absolute risk difference, 0.1 percentage points; $95 \%$ confidence interval [CI], -5.7 to $5.9 ; \mathrm{P}=0.97$ ) (Table 2). There were no significant differences in the cause of death (Table S9 in the Supplementary Appendix). No significant differences in secondary outcomes were observed (Tables 2 and 3). Kaplan-Meier survival plots showed no between-group difference in mortality rates over time (hazard ratio, 1.02; 95\% CI, 0.65 to 1.59 ; $\mathrm{P}=0.94$ ) (Fig. 2). Results of the as-treated and per-protocol analyses, which also showed no significant between-group differences, are reported in Tables S10 and S11, respectively, in the Supplementary Appendix.

Results of the prespecified and exploratory subgroup analyses are reported in Figures S3 and S4, respectively, in the Supplementary Appendix. There were no significant treatment-by-subgroup interactions. Analysis of 30-day mortality with stratification according to trial center did not identify 


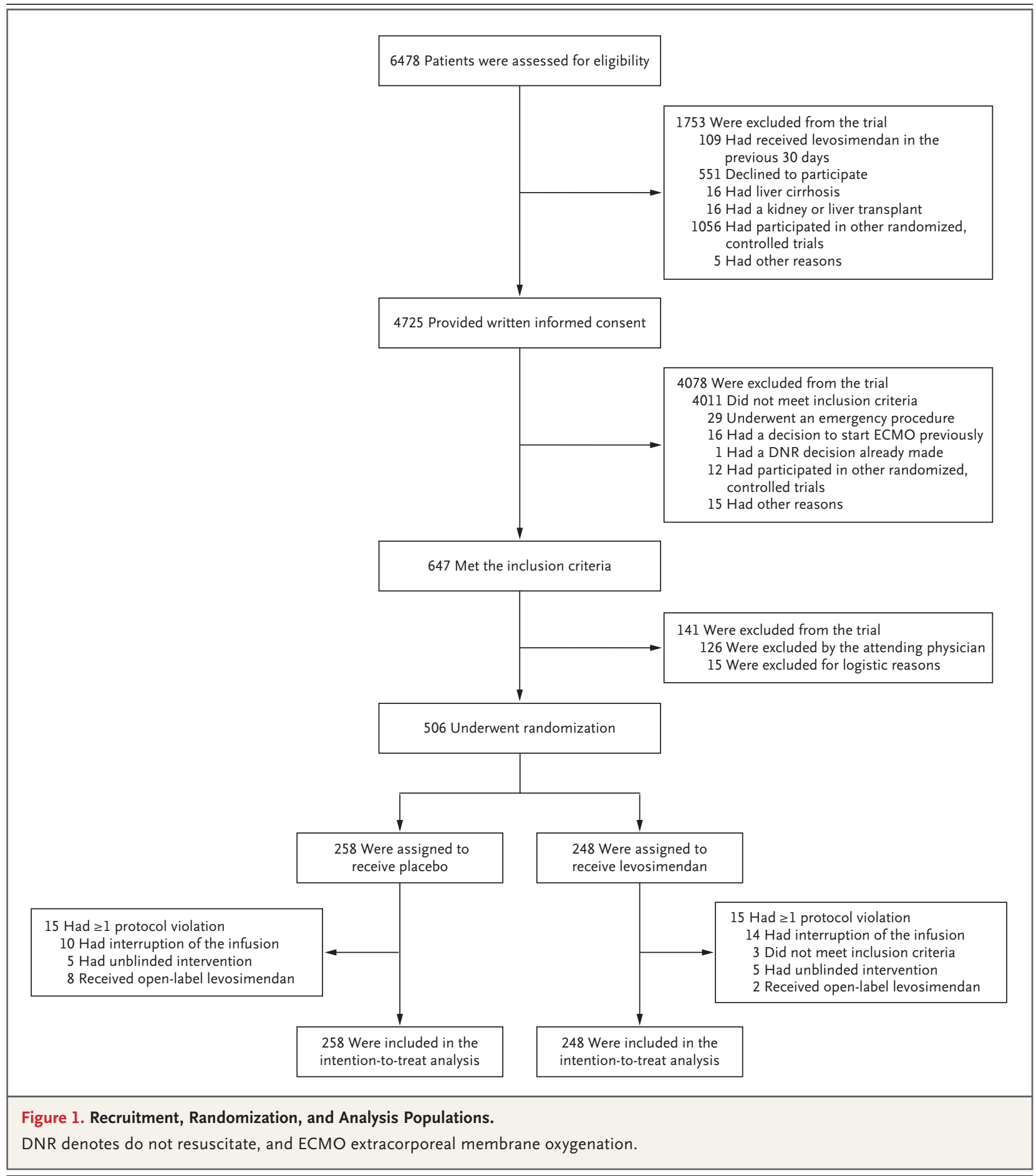

a significant interaction. Results of univariate and multivariate analyses of association of baseline variables with 30-day mortality confirmed the lack of effect of levosimendan. (Details are provided in Fig. S5 and Tables S12 and S13 in the Supplementary Appendix.)
Serious adverse events were reported in 107 of 245 patients (43.7\%) in the levosimendan group and in 131 of 254 (51.6\%) in the placebo group $(\mathrm{P}=0.08)$ (Table 3). Hypotension during the infusion was observed in 62 of 246 patients (25.2\%) in the levosimendan group and in 54 of 253 (21.3\%) 
Table 1. Characteristics of the Patients at Baseline.*

\begin{tabular}{|c|c|c|}
\hline Characteristic & $\begin{array}{l}\text { Levosimendan } \\
\text { (N=248) }\end{array}$ & $\begin{array}{c}\text { Placebo } \\
(\mathrm{N}=258)\end{array}$ \\
\hline \multicolumn{3}{|l|}{ Age $-y r$} \\
\hline Median & 66 & 66 \\
\hline Interquartile range & $58-74$ & $58-72$ \\
\hline Female sex - no. (\%) & 89 (35.9) & $90(34.9)$ \\
\hline \multicolumn{3}{|l|}{ Weight - kg } \\
\hline Median & 74 & 75 \\
\hline Interquartile range & $65-83$ & $67-86$ \\
\hline Height $-\mathrm{cm}$ & $167 \pm 8.3$ & $168 \pm 9.0$ \\
\hline \multicolumn{3}{|l|}{ Body-mass index } \\
\hline Median & 26 & 27 \\
\hline Interquartile range & $24-30$ & $24-30$ \\
\hline Previous cardiac surgery — no./total no. (\%) & $44 / 244(18.0)$ & $35 / 256(13.7)$ \\
\hline Myocardial infarction — no./total no. (\%) & $105 / 247(42.5)$ & $90 / 258(34.9)$ \\
\hline Atrial fibrillation - no./total no. (\%) & $73 / 247(29.6)$ & $81 / 258(31.4)$ \\
\hline Ongoing cardiogenic shock — no./total no. (\%)† & $6 / 247(2.4)$ & $7 / 258(2.7)$ \\
\hline \multicolumn{3}{|l|}{ NYHA classification - no./total no. (\%) } \\
\hline I & $19 / 241(7.9)$ & $20 / 249(8.0)$ \\
\hline II & $72 / 241(29.9)$ & $86 / 249(34.5)$ \\
\hline III & $133 / 241(55.2)$ & $127 / 249(51.0)$ \\
\hline IV & $17 / 241(7.1)$ & $16 / 249(6.4)$ \\
\hline COPD - no./total no. (\%) & $33 / 246(13.4)$ & $33 / 256(12.9)$ \\
\hline History of stroke or TIA — no./total no. (\%) & $18 / 247(7.3)$ & $19 / 257(7.4)$ \\
\hline Peripheral vascular disease - no./total no. (\%) & $26 / 247(10.5)$ & $42 / 257(16.3)$ \\
\hline Diabetes - no./total no. (\%) & $49 / 247(19.8)$ & $61 / 257(23.7)$ \\
\hline \multicolumn{3}{|l|}{ Left ventricular ejection fraction } \\
\hline Median (interquartile range) — $\%$ & $50(37-59)$ & $50(40-60)$ \\
\hline \multicolumn{3}{|l|}{ Distribution — no./total no. (\%) } \\
\hline$<25 \%$ & $11 / 238(4.6)$ & $11 / 251(4.4)$ \\
\hline $25-40 \%$ & $53 / 238(22.3)$ & $43 / 251(17.1)$ \\
\hline$>40 \%$ & $174 / 238(73.1)$ & $197 / 251(78.5)$ \\
\hline \multicolumn{3}{|l|}{ Preoperative medical therapy — no./total no. (\%) } \\
\hline Angiotensin-receptor blocker & $33 / 244(13.5)$ & $35 / 257(13.6)$ \\
\hline ACE inhibitor & $101 / 244(41.4)$ & $111 / 257(43.2)$ \\
\hline Diuretic & $157 / 244(64.3)$ & $162 / 257(63.0)$ \\
\hline Digoxin & $21 / 244(8.6)$ & $19 / 257(7.4)$ \\
\hline Beta-blocker & $153 / 244(62.7)$ & $159 / 257$ (61.9) \\
\hline Nitrate & $44 / 243(18.1)$ & $49 / 257(19.1)$ \\
\hline Amiodarone & $15 / 244(6.1)$ & $24 / 257(9.3)$ \\
\hline Ivabradine & $6 / 244(2.5)$ & $3 / 257(1.2)$ \\
\hline Ranolazine & $4 / 244$ (1.6) & $1 / 257(0.4)$ \\
\hline
\end{tabular}




\begin{tabular}{|c|c|c|}
\hline Characteristic & $\begin{array}{l}\text { Levosimendan } \\
\quad(\mathrm{N}=\mathbf{2 4 8})\end{array}$ & $\begin{array}{l}\text { Placebo } \\
(\mathrm{N}=258)\end{array}$ \\
\hline \multicolumn{3}{|l|}{ Inclusion criteria - no. (\%) } \\
\hline Preoperative left ventricular ejection fraction $<25 \%$ & $11(4.4)$ & $11(4.3)$ \\
\hline Intraaortic balloon pump & $50(20.2)$ & $44(17.1)$ \\
\hline $\begin{array}{l}\text { High doses of inotropes received for weaning from cardio- } \\
\text { pulmonary bypass }\end{array}$ & $33(13.3)$ & $28(10.9)$ \\
\hline High doses of inotropes administered in ICU & $154(62.1)$ & $175(67.8)$ \\
\hline
\end{tabular}

* Plus-minus values are means \pm SD. There were no significant between-group differences in the characteristics listed here. Percentages may not sum to 100 because of rounding. Data were missing as follows: on age, for 1 patient in the placebo group; on weight, height, and body-mass index (the weight in kilograms divided by the square of the height in meters) for 1 in the levosimendan group; and on left ventricular ejection fraction for 10 in the levosimendan group and for 7 in the placebo group. ACE denotes angiotensin-converting enzyme, COPD chronic obstructive pulmonary disease, ICU intensive care unit, NYHA New York Heart Association, and TIA transient ischemic attack.

$\uparrow$ Ongoing cardiogenic shock was defined as a state of end-organ hypoperfusion due to cardiac failure. The definition included the following hemodynamic variables: persistent hypotension (systolic blood pressure of 80 to $90 \mathrm{~mm} \mathrm{Hg}$ or a mean arterial pressure that was $30 \mathrm{~mm} \mathrm{Hg}$ lower than the baseline value) with a severe reduction in cardiac index $(<1.8$ liters per minute per square meter of body-surface area without support or 2.0 to 2.2 liters per minute per square meter with support) and adequate or elevated filling pressure (e.g., a left ventricular end-diastolic pressure of $>18 \mathrm{~mm}$ $\mathrm{Hg}$ or a right ventricular end-diastolic pressure of $>10$ to $15 \mathrm{~mm} \mathrm{Hg}$ ), as measured with a pulmonary-artery catheter or assessed by means of echocardiography. ${ }^{22}$

$\ddagger$ The inclusion criteria were not mutually exclusive. We list the first single criterion that led to qualification for the trial.

in the placebo group $(\mathrm{P}=0.31)$. There were 89 cases of arrhythmias, with no significant difference between the two groups.

\section{DISCUSSION}

In this multicenter, randomized, double-blind, placebo-controlled trial involving patients who required hemodynamic support after cardiac surgery, the administration of levosimendan was not associated with lower 30-day mortality than placebo. There was also no significant between-group difference in mortality in any subgroup. No significant difference was seen between the levosimendan group and the placebo group in the incidence of hypotension or arrhythmias.

Previous meta-analyses of randomized, controlled trials ${ }^{8-11}$ showed a higher rate of survival with levosimendan than with other treatment regimens among patients undergoing cardiac surgery. These findings were not confirmed in our trial. A benefit of levosimendan with regard to survival was also not shown in the LEVO-CTS (Levosimendan in Patients with Left Ventricular Systolic Dysfunction Undergoing Cardiac Surgery Requiring Cardiopulmonary Bypass) trial, the results of which have been published in the Journal. ${ }^{23} \mathrm{~A}$ similar pattern of positive results from small randomized trials and meta-analyses of randomized trials of levo- simendan, $, 8,10,24$ contradicted by a subsequent pivotal trial, has been observed in patients with severe sepsis $^{25}$ or heart failure. ${ }^{26,27}$

Cardiac surgery was considered to be the most promising context for observing a beneficial effect of levosimendan, owing to the transient nature of postoperative myocardial dysfunction..$^{28,29}$ Myocardial stunning accounts for the majority of cases of perioperative heart failure,,$^{2,28,29}$ and usually the heart recovers within 24 to 48 hours. Because of its pharmacologic characteristics (increase in cardiac output with little increase in myocardial oxygen consumption), levosimendan appeared to be the ideal inotropic agent to support heart function in such patients. However, in our trial, levosimendan did not result in lower mortality than placebo, nor did it improve other relevant outcomes in this context. Our findings do not support the administration of levosimendan in addition to standard care in the management of cardiac dysfunction after cardiac surgery.

Our trial differs from previous trials in cardiac surgery, which mostly investigated the use of levosimendan in patients undergoing coronaryartery bypass grafting (CABG). Less than half our patients underwent CABG, and a similar proportion underwent mitral-valve surgery. Thus, it is possible that perioperative cardiovascular dysfunction may have different pathophysiological features in 


\begin{tabular}{|c|c|c|c|c|}
\hline Outcome & $\begin{array}{l}\text { Levosimendan } \\
\quad(N=248)\end{array}$ & $\begin{array}{l}\text { Placebo } \\
(\mathrm{N}=258)\end{array}$ & $\begin{array}{l}\text { Difference } \\
(95 \% \mathrm{Cl}) \grave{i}\end{array}$ & P Value \\
\hline \multicolumn{5}{|l|}{ Primary outcome } \\
\hline 30-Day mortality — no. (\%) & $32(12.9)$ & $33(12.8)$ & $0.1(-5.7$ to 5.9$)$ & 0.97 \\
\hline \multicolumn{5}{|l|}{ Secondary outcomes } \\
\hline \multicolumn{5}{|l|}{$\begin{array}{l}\text { Acute kidney injury, according to RIFLE criteria } \\
\text { — no./total no. (\%) } \ddagger\end{array}$} \\
\hline Risk & $41 / 247(16.6)$ & $55 / 258(21.3)$ & $-4.7(-11.5$ to 2.1$)$ & 0.18 \\
\hline Injury & $26 / 247(10.5)$ & $27 / 258(10.5)$ & $0.1(-5.3$ to 5.4$)$ & 0.98 \\
\hline Failure & $17 / 247(6.9)$ & $22 / 258(8.5)$ & $-1.6(-6.3$ to 3.0$)$ & 0.49 \\
\hline Renal-replacement therapy — no. (\%) & $24(9.7)$ & $33(12.8)$ & $-3.1(-8.6$ to 2.4$)$ & 0.27 \\
\hline Death or renal-replacement therapy — no. (\%) & $42(16.9)$ & $49(19.0)$ & $-2.1(-8.7$ to 4.6$)$ & 0.55 \\
\hline \multicolumn{5}{|l|}{ Duration of mechanical ventilation $-\mathrm{hr}$} \\
\hline Median & 19 & 21 & $-2(-5$ to 1$)$ & 0.48 \\
\hline Interquartile range & 14 to 40 & 14 to 41 & & \\
\hline \multicolumn{5}{|l|}{ Duration of ICU stay $-\mathrm{hr}$} \\
\hline Median & 72 & 84 & $-12(-21$ to 2$)$ & 0.08 \\
\hline Interquartile range & 46 to 114 & 48 to 139 & & \\
\hline \multicolumn{5}{|l|}{ Duration of hospital stay - days } \\
\hline Median & 14 & 14 & $0(-1$ to 2$)$ & 0.39 \\
\hline Interquartile range & 8 to 21 & 9 to 21 & & \\
\hline Need for open-label levosimendan — no. (\%) & $2(0.8)$ & $8(3.1)$ & $-2.3(-4.7$ to 0.1$)$ & 0.11 \\
\hline $\begin{array}{l}\text { Interruption of infusion due to adverse events } \\
\qquad \text { no./total no. (\%) }\end{array}$ & $9 / 236(3.8)$ & $4 / 246(1.6)$ & $2.2(-0.7$ to 5.1$)$ & 0.17 \\
\hline
\end{tabular}

* Data were missing as follows: on duration of mechanical ventilation for six patients in the levosimendan group and for four in the placebo group; on duration of ICU stay for four and three, respectively; and on duration of hospital stay for three in each group.

$\dagger$ Differences between percents are presented in percentage points and may not sum as expected because of rounding. Differences in other variables are presented in the units shown in the table.

$¥$ Acute kidney injury was assessed with the use of a five-category scoring system to evaluate risk, injury, failure, loss, and end-stage kidney injury (RIFLE). ${ }^{17}$ Risk was defined as an increase in the serum creatinine level of at least 1.5 times the baseline value, a decrease in the glomerular filtration rate (GFR) of more than $25 \%$ from baseline, or a urine output of less than $0.5 \mathrm{ml}$ per kilogram of body weight per hour for 6 hours. Injury was defined as an increase in the serum creatinine level of at least 2 times the baseline value, a decrease in the GFR of more than $50 \%$ from baseline, or a urine output of less than $0.5 \mathrm{ml}$ per kilogram per hour for 12 hours. Failure was defined as an increase in the serum creatinine level of at least 3 times the baseline value, a serum creatinine level of at least $4 \mathrm{mg}$ per deciliter, a decrease in the GFR of more than $75 \%$ from baseline, a urine output of less than $0.3 \mathrm{ml}$ per kilogram per hour for 24 hours, or anuria for 12 hours.

these patients and hence result in a different response to levosimendan. However, in a prespecified subgroup analysis, we found no influence of the type of surgery on outcome.

Several previous studies of levosimendan in cardiac surgery, including the LEVO-CTS trial, ${ }^{23}$ focused on patients with reduced preoperative ejection fraction. ${ }^{11}$ In contrast, we enrolled patients with ongoing myocardial dysfunction requiring inotropic support. Although subgroup analyses involving patients with reduced ejection fraction at baseline did not show any beneficial effect of levosimendan in our trial, there were too few such patients for us to draw conclusions.

The dose of levosimendan that was used in our trial differs from that used in other studies. In most previous trials, a loading dose was administered, and in all previous trials, an infusion of at least $0.1 \mu \mathrm{g}$ per kilogram per minute was used. ${ }^{11,30}$ In our trial, we did not use a loading dose, and the infusion of levosimendan was started at $0.05 \mu \mathrm{g}$ per kilogram per minute to avoid hypotension. 
Table 3. Additional Clinical and Safety Outcomes.

\begin{tabular}{|c|c|c|c|c|}
\hline Outcome & $\begin{array}{l}\text { Levosimendan } \\
\quad(N=248)\end{array}$ & $\begin{array}{l}\text { Placebo } \\
(\mathrm{N}=258)\end{array}$ & $\begin{array}{l}\text { Difference } \\
(95 \% \mathrm{Cl}) *\end{array}$ & P Value \\
\hline \multicolumn{5}{|l|}{ Clinical outcomes } \\
\hline ECMO - no./total no. (\%) & $3 / 245(1.2)$ & $2 / 257(0.8)$ & $0.4(-1.3$ to 2.2$)$ & 0.68 \\
\hline Ventricular assist device - no./total no. (\%) & $1 / 244(0.4)$ & $1 / 257(0.4)$ & $0.0(-1.1$ to 1.1$)$ & 0.99 \\
\hline Myocardial infarction — no./total no. (\%) & $14 / 243(5.8)$ & $15 / 257(5.8)$ & $-0.1(-4.2$ to 4.0$)$ & 0.99 \\
\hline \multicolumn{5}{|l|}{ Neurologic damage — no./total no. (\%) $†$} \\
\hline Type 1 & $11 / 247(4.5)$ & $9 / 258(3.5)$ & $1.0(-2.4$ to 4.4$)$ & 0.58 \\
\hline Type 2 & $30 / 247(12.1)$ & $37 / 258(14.3)$ & $-2.2(-8.1$ to 3.7$)$ & 0.47 \\
\hline Need for tracheostomy — no./total no. (\%) & $17 / 247(6.9)$ & $14 / 255(5.5)$ & $1.4(-2.8$ to 5.6$)$ & 0.52 \\
\hline Sepsis - no./total no. (\%) & $16 / 246(6.5)$ & $17 / 255(6.7)$ & $-0.2(-4.5$ to 4.2$)$ & 0.93 \\
\hline Severe sepsis — no./total no. (\%) & $9 / 245(3.7)$ & $13 / 255(5.1)$ & $-1.4(-5.0$ to 2.1$)$ & 0.43 \\
\hline Septic shock - no./total no. (\%) & $6 / 245(2.4)$ & $10 / 255(3.9)$ & $-1.5(-4.6$ to 1.6$)$ & 0.35 \\
\hline Mediastinitis — no./total no. (\%) & $1 / 242(0.4)$ & $4 / 253(1.6)$ & $-1.2(-2.9$ to 0.6$)$ & 0.37 \\
\hline Pneumonia — no./total no. (\%) & $15 / 243(6.2)$ & $15 / 253(5.9)$ & $0.2(-4.0$ to 4.4$)$ & 0.91 \\
\hline \multicolumn{5}{|l|}{ Transfusion in operating room or ICU } \\
\hline \multicolumn{5}{|l|}{ Red-cell transfusion } \\
\hline No. of patients (\%) & $125(50.4)$ & $141(54.7)$ & $-4.2(-12.9$ to 4.4$)$ & 0.34 \\
\hline \multicolumn{5}{|l|}{ Units per patient } \\
\hline Median & 2.0 & 3.0 & $-1.0(-1.0$ to 1.0$)$ & 0.21 \\
\hline Interquartile range & 2.0 to 4.0 & 2.0 to 5.0 & & \\
\hline \multicolumn{5}{|l|}{ Fresh-frozen plasma transfusion } \\
\hline No. of patients (\%) & $72(29.0)$ & $90(34.9)$ & $-5.9(-14.0$ to 2.2$)$ & 0.16 \\
\hline \multicolumn{5}{|l|}{ Units per patient } \\
\hline Median & 3.0 & 3.0 & $0.0(-1.0$ to 1.0$)$ & 0.40 \\
\hline Interquartile range & 2.0 to 4.0 & 2.0 to 7.0 & & \\
\hline \multicolumn{5}{|l|}{ Platelet transfusion } \\
\hline No. of patients (\%) & $17(6.9)$ & $26(10.1)$ & $-3.1(-8.1$ to 1.6$)$ & 0.19 \\
\hline \multicolumn{5}{|l|}{ Units per patient } \\
\hline Median & 1.0 & 1.0 & $0.0(-1.0$ to 1.0$)$ & 0.37 \\
\hline Interquartile range & 1.0 to 2.0 & 1.0 to 3.0 & & \\
\hline \multicolumn{5}{|l|}{ Death — no./total no. (\%) } \\
\hline In the ICU & $24 / 248(9.7)$ & $19 / 258(7.4)$ & $2.3(-2.6$ to 7.2$)$ & 0.35 \\
\hline In the hospital & $31 / 248(12.5)$ & $31 / 258(12.0)$ & $0.5(-5.2$ to 6.2$)$ & 0.87 \\
\hline At 180 days & $38 / 248(15.3)$ & $39 / 254(15.4)$ & $0.0(-6.3$ to 6.3$)$ & 0.99 \\
\hline \multicolumn{5}{|l|}{ Safety outcomes } \\
\hline Hypotension during infusion - no./total no. (\%) & $62 / 246(25.2)$ & $54 / 253(21.3)$ & $3.9(-3.6$ to 11.3$)$ & 0.31 \\
\hline Managed with vasoconstrictors & $43 / 56(76.8)$ & $40 / 53(75.5)$ & $1.3(-14.7$ to 17.3$)$ & 0.87 \\
\hline Managed with dose reduction & $26 / 50(52.0)$ & $19 / 45(42.2)$ & $10.7(-9.2$ to 30.6$)$ & 0.29 \\
\hline \multicolumn{5}{|l|}{ Arrhythmias during infusion — no./total no. (\%) } \\
\hline Supraventricular & $35 / 246(14.2)$ & $43 / 254(16.9)$ & $-2.7(-9.1$ to 3.7$)$ & 0.41 \\
\hline Ventricular & $4 / 246(1.6)$ & $7 / 254(2.8)$ & $-1.1(-3.7$ to 1.4$)$ & 0.55 \\
\hline \multicolumn{5}{|l|}{ Serious adverse event — no./total no. (\%) } \\
\hline Considered by investigator to be due to trial regimen & $0 / 244$ & $0 / 256$ & - & - \\
\hline Anyț & $107 / 245(43.7)$ & $131 / 254(51.6)$ & $-7.9(-16.6$ to 0.8$)$ & 0.08 \\
\hline
\end{tabular}

* Differences between percent values are presented in percentage points and may not sum as expected because of rounding. Differences in other variables are presented in the units shown in the table.

$\dagger$ Neurologic damage type 1 was defined as death due to stroke or hypoxic encephalopathy, nonfatal stroke, transient ischemic attack, or stupor or coma at the time of discharge. ${ }^{18}$ Neurologic damage type 2 was defined as new deterioration in intellectual function, confusion, agitation, disorientation, memory deficit, or seizure without evidence of focal injury. ${ }^{18}$

$\lceil$ This outcome included a composite of myocardial infarction, acute kidney injury (any stage), neurologic damage type 1 or type 2 , septic shock, pneumonia, and mediastinitis. 


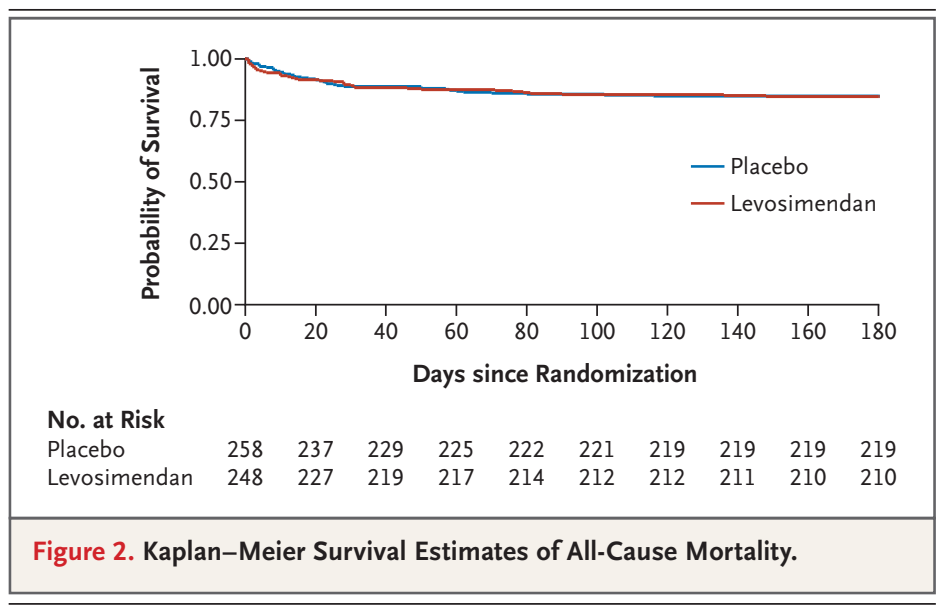

Although attending physicians were permitted to increase the infusion dose, the mean infusion dose of levosimendan that was administered in our trial was $0.07 \mu \mathrm{g}$ per kilogram per minute. We chose this conservative approach because loading doses and high-dose infusions have been associated with hypotension and a less marked beneficial effect on survival. ${ }^{10}$ In a recent trial involving patients with sepsis, an infusion dose of $0.2 \mu \mathrm{g}$ per kilogram per minute was associated with a higher incidence of hypotension and arrhythmias and a higher rate and longer duration of norepinephrine infusion than placebo ${ }^{25}$ - effects that did not occur in our trial. At the lower dose we used, we found no difference in cardiac index over time between the two groups (although cardiac index was not systematically recorded in our patients). However, a hemodynamic effect of levosimendan is suggested by the greater number of attempts to increase the dose in the placebo group and the higher mean dose in the placebo group.
Our trial has some limitations. First, the trial was interrupted early on the grounds of futility for the primary outcome (30-day mortality). This situation may have increased the potential for type II error in the secondary outcomes. Second, despite the signals of a hemodynamic effect of levosimendan, we cannot rule out the possibility that higher doses might have been effective in reducing mortality, although higher doses might also have increased the risk of adverse effects such as hypotension and arrhythmias. Third, we investigated a mixed population of patients who were undergoing different cardiac surgical operations, including a few patients (2.2\%) without cardiopulmonary bypass. However, none of the subgroup analyses suggested a benefit in association with levosimendan treatment. Fourth, we did not systematically collect cardiac-output data, which could have helped us understand and interpret the results of the trial. Owing to the fact that our enrollment criteria were based mainly on the need for hemodynamic support, we may have enrolled some patients who did not have underlying severe cardiac dysfunction.

In conclusion, in patients with perioperative left ventricular dysfunction requiring hemodynamic support after cardiac surgery, a low-dose infusion of levosimendan did not result in lower 30-day mortality than placebo nor did it positively affect any secondary-outcome measures as compared with placebo.

Supported by a grant (RF-2009-1519827) from the Italian Ministry of Health.

Disclosure forms provided by the authors are available with the full text of this article at NEJM.org.

We thank Ms. Paola Zuppelli for administrative assistance with the organization and coordination of the trial, and the physicians and nurses of all the participating departments for assistance.

The authors' full names and academic degrees are as follows: Giovanni Landoni, M.D., Vladimir V. Lomivorotov, M.D., Ph.D., Gabriele Alvaro, M.D., Rosetta Lobreglio, M.D., Antonio Pisano, M.D., Fabio Guarracino, M.D, Maria G. Calabrò, M.D., Evgeny V. Grigoryev, M.D., Ph.D., Valery V. Likhvantsev, M.D., Ph.D., Marcello F. Salgado-Filho, M.D., Ph.D., Alessandro Bianchi, M.D., Vadim V. Pasyuga, M.D., Massimo Baiocchi, M.D., Federico Pappalardo, M.D., Fabrizio Monaco, M.D., Vladimir A. Boboshko, M.D., Ph.D., Marat N. Abubakirov, M.D., Bruno Amantea, M.D., Rosalba Lembo, M.Sc., Luca Brazzi, M.D., Ph.D., Luigi Verniero, M.D., Pietro Bertini, M.D., Anna M. Scandroglio, M.D., Tiziana Bove, M.D., Alessandro Belletti, M.D., Maria G. Michienzi, M.D., Dmitriy L. Shukevich, M.D., Ph.D., Tatiana S. Zabelina, M.D., Rinaldo Bellomo, M.D., Ph.D., and Alberto Zangrillo, M.D.

The authors' affiliations are as follows: the Department of Anesthesia and Intensive Care, San Raffaele Scientific Institute (G.L., M.G.C., F.P., F.M., R. Lembo, A.M.S., T.B., A. Belletti, A.Z.), and Vita-Salute San Raffaele University (G.L., A.Z.), Milan, the Department of Anesthesia and Intensive Care, Azienda Ospedaliero-Universitaria Mater Domini Germaneto, Catanzaro (G.A., B.A., M.G.M.), the Department of Anesthesia and Intensive Care, Azienda Ospedaliero-Universitaria Città della Salute e della Scienza (R. Lobreglio, L.B.), the Department of Cardiovascular Anesthesia and Intensive Care, Azienda Ospedaliera Ordine Mauriziano (A. Bianchi), and the Department of Surgical Sciences, University of Turin (L.B.), Turin, the Division of Cardiac Anesthesia and Intensive Care Unit, Azienda Ospedaliera di Rilievo Nazionale dei Colli-Monaldi Hospital, Naples (A.P., L.V.), Division of Cardiothoracic and Vascular Anesthesia and Intensive Care, the Department of Anesthesia and Critical Care Medicine, Azienda Ospedaliero-Universitaria Pisana, Pisa (F.G., P.B.), and the Department of Cardiothoracic and Vascular Anesthesia and Intensive Care, University Hospital Policlinico S. Orsola, Bologna 
(M.B.) - all in Italy; the Department of Anesthesiology and Intensive Care, State Research Institute of Circulation Pathology, Novosibirsk (V.V. Lomivorotov, V.A.B., M.N.A.), the Department of Anesthesiology and Intensive Care, State Research Institute for Complex Issues of Cardiovascular Disease, Kemerovo (E.V.G., D.L.S.), the Department of Anesthesiology and Intensive Care, Moscow Regional Clinical and Research Institute, Moscow (V.V. Likhvantsev, T.S.Z.), and the Department of Anesthesiology and Intensive Care, Federal Center for Cardiovascular Surgery Astrakhan, Astrakhan (V.V.P.) - all in Russia; Federal University of Juiz de Fora, Juiz de Fora, Brazil (M.F.S.-F.); and the University of Melbourne, Melbourne, VIC, Australia (R.B.).

\section{REFERENCES}

1. Mozaffarian D, Benjamin EJ, Go AS, et al. Heart disease and stroke statistics - 2016 update: a report from the American Heart Association. Circulation 2016; 133(4):e38-e360.

2. Mebazaa A, Pitsis AA, Rudiger A, et al. Clinical review: practical recommendations on the management of perioperative heart failure in cardiac surgery. Crit Care 2010;14:201.

3. Lomivorotov VV, Efremov SM, Kirov MY, Fominskiy EV, Karaskov AM. Low-car diac-output syndrome after cardiac surgery. J Cardiothorac Vasc Anesth 2017;31:291 308.

4. Maganti M, Badiwala M, Sheikh A, et al. Predictors of low cardiac output syndrome after isolated mitral valve surgery. J Thorac Cardiovasc Surg 2010;140:790-6. 5. Gillies M, Bellomo R, Doolan L, Buxton B. Bench-to-bedside review: inotropic drug therapy after adult cardiac surgery - a systematic literature review. Crit Care 2005;9:266-79.

6. Thackray S, Easthaugh J, Freemantle $\mathrm{N}$, Cleland JG. The effectiveness and relative effectiveness of intravenous inotropic drugs acting through the adrenergic pathway in patients with heart failure a meta-regression analysis. Eur J Heart Fail 2002;4:515-29.

7. Shahin J, DeVarennes B, Tse CW, Amarica DA, Dial S. The relationship be tween inotrope exposure, six-hour postoperative physiological variables, hospital mortality and renal dysfunction in patients undergoing cardiac surgery. Crit Care 2011;15:R162.

8. Pollesello P, Parissis J, Kivikko M, Harjola VP. Levosimendan meta-analyses: is there a pattern in the effect on mortality? Int J Cardiol 2016;209:77-83.

9. Landoni G, Mizzi A, Biondi-Zoccai G, et al. Reducing mortality in cardiac surgery with levosimendan: a meta-analysis of randomized controlled trials. J Cardiothorac Vasc Anesth 2010;24:51-7.

10. Landoni G, Biondi-Zoccai G, Greco $\mathrm{M}$, et al. Effects of levosimendan on mor- tality and hospitalization: a meta-analysis of randomized controlled studies. Crit Care Med 2012;40:634-46.

11. Harrison RW, Hasselblad V, Mehta RH, Levin R, Harrington RA, Alexander $\mathrm{JH}$. Effect of levosimendan on survival and adverse events after cardiac surgery: a meta-analysis. J Cardiothorac Vasc Anesth 2013;27:1224-32.

12. Greco T, Calabrò MG, Covello RD, et al. A Bayesian network meta-analysis on the effect of inodilatory agents on mortality. Br J Anaesth 2015;114:746-56

13. Papp Z, Édes I, Fruhwald S, et al. Levosimendan: molecular mechanisms and clinical implications: consensus of experts on the mechanisms of action of levosimendan. Int J Cardiol 2012;159:82-7.

14. Nieminen MS, Fruhwald S, Heunks LM, et al. Levosimendan: current data, clinical use and future development. Heart Lung Vessel 2013;5:227-45.

15. Zangrillo A, Alvaro G, Pisano A, et al. A randomized controlled trial of levosimendan to reduce mortality in high-risk cardiac surgery patients (CHEETAH): rationale and design. Am Heart J 2016;177:66-73.

16. Ford I, Norrie J. Pragmatic trials. N Engl J Med 2016;375:454-63.

17. Bellomo R, Ronco C, Kellum JA, Mehta RL, Palevsky P. Acute renal failure definition, outcome measures, animal models, fluid therapy and information technology needs: the Second International Consensus Conference of the Acute Dialysis Quality Initiative (ADQI) Group. Crit Care 2004;8:R204-R212.

18. Roach GW, Kanchuger M, Mangano $\mathrm{CM}$, et al. Adverse cerebral outcomes after coronary bypass surgery. N Engl J Med 1996;335:1857-63.

19. Ridgeon E, Bellomo R, Myburgh J, et al. Validation of a classification system for causes of death in critical care: an assessment of inter-rater reliability. Crit Care Resusc 2016;18:50-4.

20. DeMets DL, Pocock SJ, Julian DG. The agonising negative trend in monitoring of clinical trials. Lancet 1999;354:1983-8.
21. Newcombe RG. Interval estimation for the difference between independent proportions: comparison of eleven methods. Stat Med 1998;17:873-90.

22. Reynolds HR, Hochman JS. Cardiogenic shock: current concepts and improving outcomes. Circulation 2008;117: 686-97.

23. Mehta RH, Leimberger JD, van Diepen $S$, et al. Levosimendan in patients with left ventricular dysfunction undergoing cardiac surgery. N Engl J Med. DOI: 10.1056/NEJMoa1616218.

24. Zangrillo A, Putzu A, Monaco F, et al. Levosimendan reduces mortality in patients with severe sepsis and septic shock: a meta-analysis of randomized trials. J Crit Care 2015;30:908-13.

25. Gordon AC, Perkins GD, Singer M, et al. Levosimendan for the prevention of acute organ dysfunction in sepsis. $\mathrm{N}$ Engl J Med 2016;375:1638-48.

26. Mebazaa A, Nieminen MS, Packer M, et al. Levosimendan vs dobutamine for patients with acute decompensated heart failure: the SURVIVE randomized trial. JAMA 2007;297:1883-91.

27. Packer M, Colucci W, Fisher L, et al. Effect of levosimendan on the shortterm clinical course of patients with acutely decompensated heart failure. JACC Heart Fail 2013;1:103-11.

28. Rudiger A, Businger F, Streit $M$, Schmid ER, Maggiorini M, Follath F. Presentation and outcome of critically ill medical and cardiac-surgery patients with acute heart failure. Swiss Med Wkly 2009;139: 110-6.

29. St André AC, DelRossi A. Hemodynamic management of patients in the first 24 hours after cardiac surgery. Crit Care Med 2005;33:2082-93.

30. Toller W, Heringlake M, Guarracino F, et al. Preoperative and perioperative use of levosimendan in cardiac surgery: European expert opinion. Int J Cardiol 2015; 184:323-36.

Copyright (c) 2017 Massachusetts Medical Society. 